\title{
Paper Multiple-ring Circular Array for Ground-Penetrating Radar Applications: Basic Ideas and Preliminary Results
}

\author{
Roberto Vescovo ${ }^{1}$ and Lara Pajewski ${ }^{2}$ \\ ${ }^{1}$ Department of Engineering and Architecture, University of Trieste, Trieste, Italy \\ ${ }^{2}$ Department of Information Engineering, Electronics and Telecommunications, Sapienza University of Rome, Rome, Italy
}

https://doi.org/10.26636/jtit.2017.120217

\begin{abstract}
In this paper, the possibility of using a multiplering circular array as an antenna array for Ground-Penetrating Radar systems is investigated. The theory behind the proposed idea is presented. The preliminary numerical results that are obtained suggest that the proposed configuration is promising. It allows achieving a wide frequency band and low dynamic range ratio of excitations, thus simplifying the feeding network. Further interesting requirements may be satisfied by exploiting a combination of deterministic and stochastic synthesis techniques to design the array.
\end{abstract}

Keywords—antenna array, ultra-wideband, Ground-Penetrating Radar.

\section{Introduction}

Antennas are a critical hardware component of a radar system, dictating its performance in terms of capability to detect targets. Nevertheless, most research efforts in the Ground-Penetrating Radar (GPR) field are focused on the applications of the technique and on the development of modeling, inversion and data-processing approaches [1], [2]. Only a limited number of studies deal with technological issues related to the design of novel systems [3]-[7], including the synthesis, optimization and characterization of new antennas [8], [9]. Even fewer are the research projects where innovative antenna arrays are developed [10].

Requirements that GPR antennas have to satisfy are somehow unique and very different than in conventional radar antennas, as GPR antennas operate in a strongly demanding environment, in close proximity to or at a limited distance from the natural or manmade investigated structure [11]. The same applies to GPR antenna arrays.

The first requirement is an ultra-wide frequency band: the radar has to transmit and receive short-duration timedomain waveforms, in the order of a few nanoseconds, the time-duration of the emitted pulses being a trade-off between the desired radar resolution and penetration depth. Additionally, GPR antennas shall have a linear phase characteristic over the whole operational frequency range, as well as predictable polarization and gain. Due to the fact that a subsurface imaging system is essentially a short-range radar, the coupling between transmitting and receiving antennas has to be low and short in time. Moreover, GPR antennas shall have quick ring-down characteristics, in order to prevent masking of targets and guarantee a good resolution. The radiation pattern shall ensure minimal interference with unwanted objects, usually present in the complex operational environment. To this aim, antennas have to provide high directivity and concentrate the electromagnetic energy into a narrow solid angle. As GPR antennas work very close to the matter or even in contact with it, changes in electrical properties of the matter should not affect strongly the antenna performance, so that a wide applicability of the radar system can be achieved. Furthermore, antennas should provide stable performance at different elevation levels. For an efficient coupling of electromagnetic waves into the ground/investigated structure, good impedance matching is necessary at the antenna/matter interface. Another important requirement regards the weight and size of the antennas: for ease of utilization and to allow a wide applicability, GPR antennas shall be light and compact.

Array of antennas can be used in GPR systems to enable a faster data collection by increasing the extension of investigated area per time unit. This can be a significant advantage in archaeological prospection, road and bridge inspection, mine detection, as well as in several other civil engineering and geoscience applications where the collection of data requires the execution of a large number of profiles. Moreover, antenna arrays allow collecting multi-offset measurements simultaneously, thereby providing additional information for a more effective imaging and characterization of the scenario under test.

Two approaches are possible to GPR array design. The simplest and most common is to conceive the array as a multichannel radar system composed of single-channel radars. Much more can be achieved, if array-design techniques are employed to synthesize the whole system. This second approach is just starting in the GPR field and is definitely promising, as it gives the possibility to fully exploit the potentiality of arrays: they can provide a high directivity by using simple elements and the capability of a steerable beam, as in smart antennas. 
Another important issue, to be considered when using multi-antenna GPR systems on irregular surfaces, is that the position of array elements has to be recorded during the surveys, by using high-precision positioning systems [12]. In this paper, which resumes and further extends the contribution presented in [13], we investigate the possibility of using a multiple-ring circular array as an antenna array for GPR systems. This configuration seems promising for achieving a wide frequency band and low dynamic range ratio of excitations, thus simplifying the feeding network. Further interesting requirements might be satisfied by exploiting a combination of deterministic and stochastic synthesis techniques to design the array.

\section{Theory of Multiple-ring Circular Array}

The concept of pattern multiplication is well known. If all antennas of an array are identical and have the same physical orientation, then the radiation (or reception) pattern of the array is given by the radiation pattern of the single antenna element multiplied by the array factor. With reference to Fig. 1, the array factor $F$ is a function of the direction of observation, specified by the unit vector $\hat{\mathbf{r}}$. It also depends on the positions of the $\mathrm{N}$ antennas in the array, $\mathbf{r}_{\mathrm{n}}$, and on the complex weights used to feed them, $a_{n}(\mathrm{n}=1, \ldots, \mathrm{N})$ :

$$
F=\sum_{\mathrm{n}=1}^{\mathrm{N}} a_{\mathrm{n}} \mathrm{e}^{j k \mathbf{r}_{\mathrm{n}} \cdot \hat{\mathbf{r}}}
$$

where $k=\frac{2 \pi}{\lambda}$ is the wave number, being $\lambda$ the wavelength.

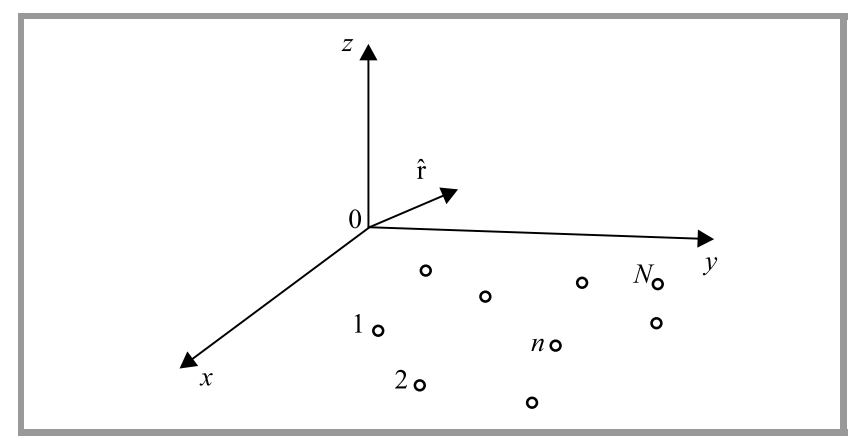

Fig. 1. Antenna array of $N$ elements in arbitrary positions.

By tailoring the geometry and feeding network of an array, its performance may be optimized to achieve desirable properties. For instance, the array pattern can be steered (i.e., the direction of maximum radiation or reception can be changed) by modifying the weights.

In a GPR antenna array, the single element will be an ultrawideband antenna (see Fig. 2). It is desirable that the overall radiation pattern of the array be "independent" of the frequency, not only the radiation pattern of the single antenna. Therefore, the array factor has to be "independent" of the frequency as well. A possible solution is a multiple

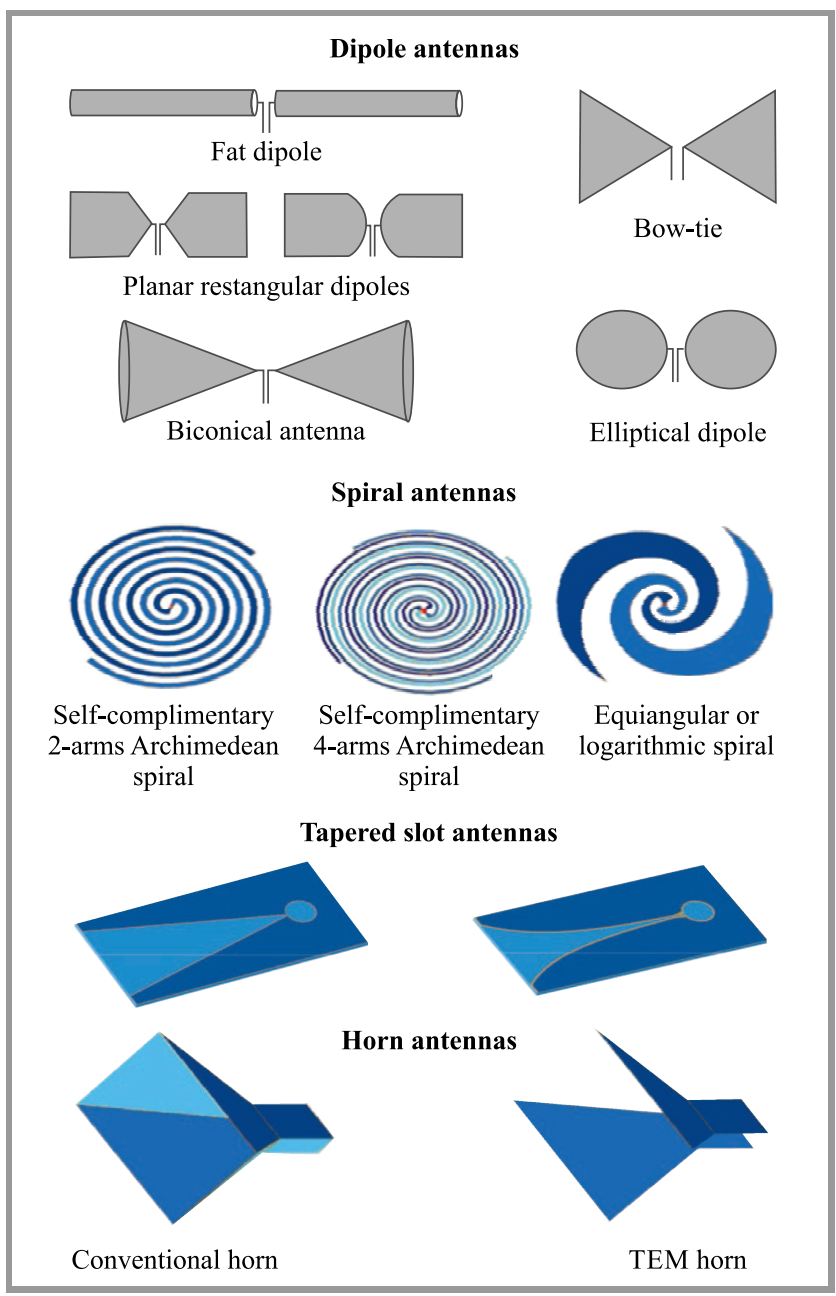

Fig. 2. Most frequently used GPR antennas.

ring circular array, schematized in Fig. 3. This is constituted by $\mathrm{M}$ concentric rings of radii $R_{\mathrm{m}}$, where each ring includes $L_{\mathrm{m}}$ equispaced antennas $(m=1, \ldots, \mathrm{M})$. The total number of antennas is:

$$
\mathrm{N}=\sum_{m=1}^{\mathrm{M}} L_{\mathrm{m}} .
$$

In a customary spherical coordinate system $(r, \theta, \phi)$ with origin in the center of the radiating rings, the array factor of radiating rings with a continuous excitation $a_{\mathrm{m}}$ (being $a_{\mathrm{m}}$ the excitation density on the $m$-th ring) has the following expression:

$$
F=\sum_{\mathrm{m}=1}^{\mathrm{M}} a_{\mathrm{m}} R_{\mathrm{m}} \int_{0}^{2 \pi} \mathrm{e}^{j R_{\mathrm{m}} k \sin \theta \cos \phi^{\prime}} \mathrm{d} \phi^{\prime} .
$$

Recalling the integral properties of the Bessel functions, it results:

$$
F=2 \pi \sum_{\mathrm{m}=1}^{\mathrm{M}} a_{\mathrm{m}} R_{\mathrm{m}} J_{0}\left(R_{\mathrm{m}} k \sin \theta\right) .
$$

In the structure that we are considering, instead, the excitation is discrete. The $\ell$-th element on the ring $\mathrm{m}(\ell=$ $\left.1, \ldots, L_{\mathrm{m}}\right)$ is located at the angular position

$$
\phi_{\ell \mathrm{m}}=\frac{2 \pi(\ell-1)}{L_{\mathrm{m}}}+\delta_{m},
$$




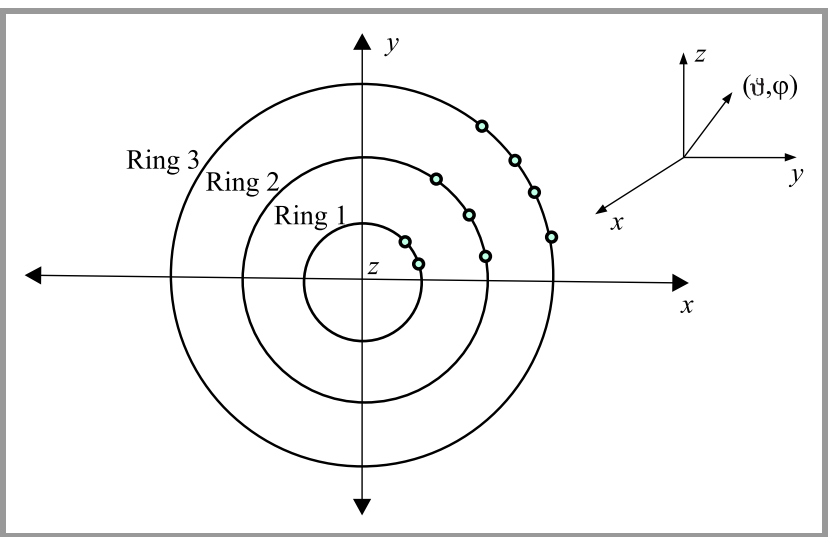

Fig. 3. Multiple ring circular array.

where $\delta_{\mathrm{m}}$ is the angular position of the first element. Let us call $a_{\ell \mathrm{m}}$ the excitation of the $\ell$-th element on the ring $\mathrm{m}$ and let us also assume that uniform excitation is adopted in each ring, i.e.:

$$
a_{\ell \mathrm{m}}=\frac{2 \pi R_{\mathrm{m}} a_{\mathrm{m}}}{L_{\mathrm{m}}} \forall \ell .
$$

Then, by using Eq. (1) and after some manipulations, the array factor is given by:

$$
F=\sum_{\mathrm{m}=1}^{\mathrm{M}} \frac{2 \pi R_{\mathrm{m}}}{L_{\mathrm{m}}} a_{\mathrm{m}} \sum_{\ell=1}^{L_{\mathrm{m}}} \mathrm{e}^{j R_{\mathrm{m}} k \sin \theta \cos \left(\phi-\phi_{\ell \mathrm{m}}\right)} .
$$

Such array factor is weakly affected by a change of the frequency, as will be illustrated in Section 3 via a numerical example.

\section{Results}

In this Section, we present a simple example of array-factor synthesis for the multiple-ring circular array. The aim of this example is to illustrate the frequency-dispersion properties of the array factor. In the example, we set $\delta_{\mathrm{m}}=0$. As a desired pattern, we choose $F_{d}(u)=\left|\operatorname{sinc}\left(\mathrm{c}_{1} \mathrm{u}\right)\right|^{\mathrm{c}_{2}}$, where $u=k \sin \theta$, and $c_{1}, c_{2}$ are given real numbers. This pattern has a maximum in $\theta=0$. By exploiting Eq. (4), we want to find the coefficients $a_{\mathrm{m}}$ that minimize the squared distance:

$$
\rho^{2}=\left\|F_{d}(u)-2 \pi \sum_{\mathrm{m}=1}^{M} a_{\mathrm{m}} R_{\mathrm{m}} J_{0}\left(R_{\mathrm{m}} u\right)\right\|^{2},
$$

where $\|f\|=\int_{0}^{2 \pi}|f(\gamma)|^{2} \mathrm{~d} \gamma$. By manipulating (6) and setting

$$
\frac{\partial \rho}{\partial a_{\mathrm{p}}}=0, \quad \mathrm{p}=1, \ldots, \mathrm{M}
$$

we obtain a system of $\mathrm{M}$ linear equations in the $\mathrm{M}$ (real) unknowns $a_{\mathrm{m}}$ :

$$
\sum_{\mathrm{m}=1}^{\mathrm{M}} T_{\mathrm{pm}} a_{\mathrm{m}}=U_{\mathrm{p}} \quad(\mathrm{p}=1, \ldots, \mathrm{M}),
$$

where $T_{\mathrm{pm}}=2 \pi R_{\mathrm{m}}<J_{0}\left(R_{\mathrm{m}} u\right), J_{0}\left(R_{\mathrm{p}} u\right)>$ and $U_{\mathrm{p}}=<F_{d}$, $J_{0}\left(R_{\mathrm{p}} u\right)>$, being $<f_{1}, f_{2}>=\int_{0}^{2 \pi} f_{1}(\gamma) f_{2}(\gamma) \mathrm{d} \gamma$ a scalar product between the real functions $f_{1}$ and $f_{2}$. The system (7) can be solved by standard techniques and the solutions $a_{\mathrm{m}}$ minimize $\rho^{2}$ in Eq. (6).

The same coefficients $a_{\mathrm{m}}$ can be used in (5) as well, to calculate the array factor of the array with $L_{\mathrm{m}}$ elements on the $\mathrm{m}$-th ring.

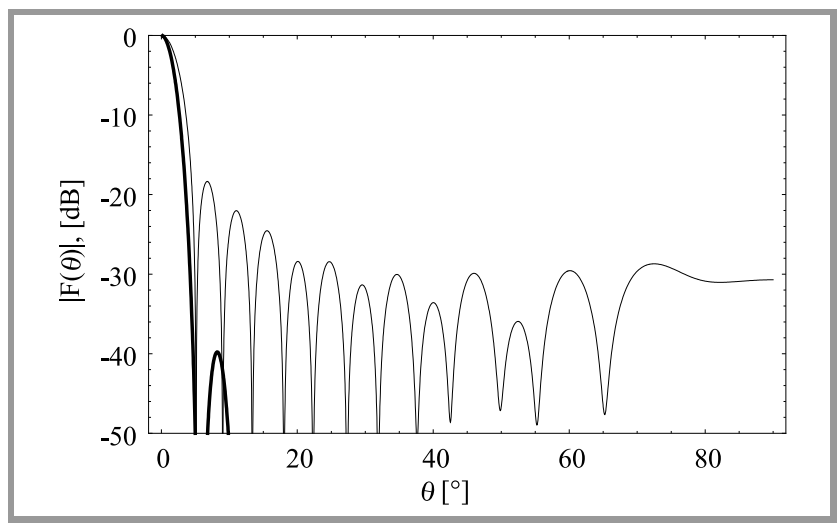

Fig. 4. Desired (thick line) and synthesized (thin line) patterns for the multiple-ring circular array with $\mathrm{M}=8$ rings described in the text, when $\phi=0$ and at the nominal frequency $f_{0}$.

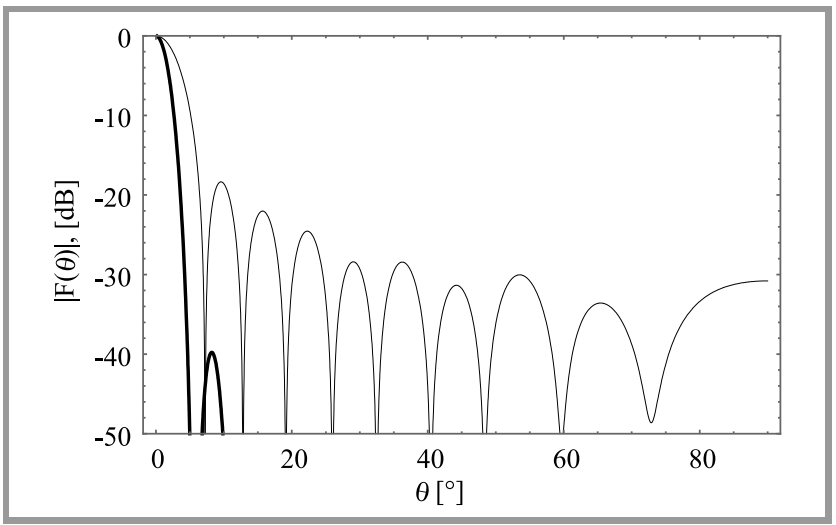

Fig. 5. Same as in Fig. 4, when the frequency is $f_{1}=0.707 f_{0}$.

In Fig. 4, the desired and synthesized patterns are plotted as a function of $\theta$ when $\phi=0$, for a multiple-ring circular array with $\mathrm{M}=8$ rings. Different cuts of the radiation pattern give similar results. The number of elements and radius of the $\mathrm{m}$-th ring are: $L_{\mathrm{m}}=5 \mathrm{~m}, R_{\mathrm{m}}=0.8 \mathrm{~m} \lambda(\mathrm{m}=1, \ldots, \mathrm{M})$. Moreover, $c_{1}=5$ and $c_{2}=3$. The dynamic range ratio, which is the ratio between the maximum and minimum excitation amplitudes of the array elements, is $D R R=1.375$. Note that a limited dynamic range ratio allows the practical realization of noncomplex feeding networks, where the number of power dividers may be kept low or the design of the feeding lines may be simplified.

Now, for the same geometry and keeping the same excitations, we modify the frequency and study how a change of frequency affects the radiation pattern.

In Fig. 5, the same as in Fig. 4 is shown, when the frequency is $f_{1}=\frac{f_{0}}{\sqrt{2}}=0.707 f_{0}$. In Fig. 6 , the same as in Fig. 4 is shown, when the frequency is $f_{2}=1.1 f_{0}$. Finally, 


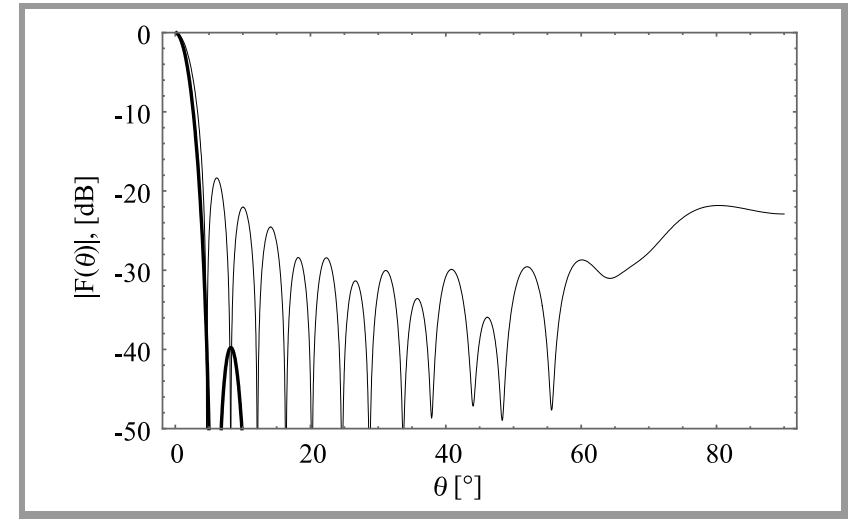

Fig. 6. Same as in Fig. 4 , when the frequency is $f_{2}=1.1 f_{0}$.

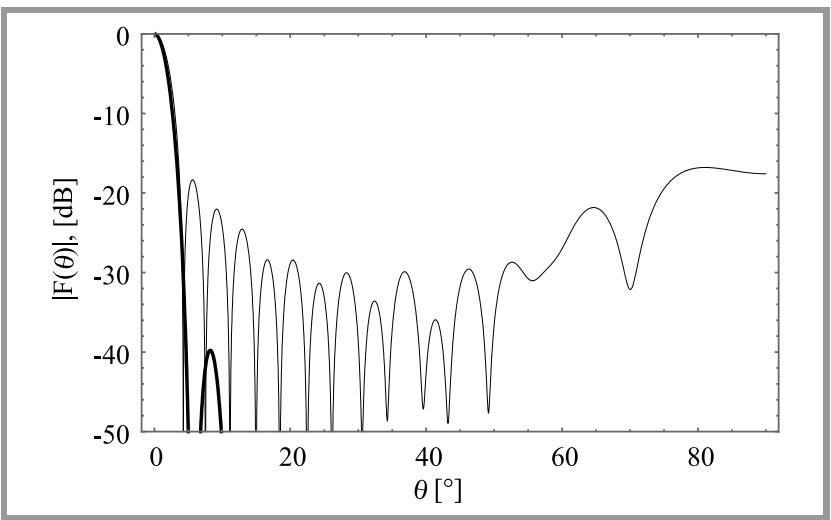

Fig. 7. Same as in Fig. 4 , when the frequency is $f_{3}=1.5 f_{0}$.

in Fig. 7, the same as in Fig. 4 is shown, when the frequency is $f_{3}=1.5 f_{0}$. Note that, in the latter case, $f_{3}-f_{0}=0.5 f_{0}$. It is apparent that the radiation properties of the array are not significantly affected by the frequency change.

\section{Conclusions and Future Work}

Usually, a multi-antenna Ground-Penetrating Radar (GPR) is simply conceived as a multi-channel radar system composed of single-channel independent systems. Much more can be achieved, if array-design techniques are employed to synthesize the overall set of antennas. Such approach is slowly starting in the GPR field and is definitely promising, as it gives the possibility to fully exploit the potentiality of arrays.

In this paper, we have suggested and investigated the multiple circular-ring array, as a possible solution for a GPR antenna array. The preliminary results that we have obtained show that our idea is promising. The proposed structure yields the possibility of a wide frequency band with a low dynamic range ratio. Moreover, the synthesis can be easily performed at a single frequency within the range of interest.

The natural continuation of this work is the design of a realistic GPR array and the testing of its performance in a realistic scenario.
For the design of the GPR array, two deterministic methods may be used, i.e. the techniques presented in [14] and [15]. The former is based on an alternate projections approach and requires a given array geometry. The latter allows an optimization of the radii of the circular rings. The method determines the array geometry starting from a dense grid of fictitious elements and reducing their number iteratively. This allows to optimize the array geometry and to meet constraints on the copolar and crosspolar patterns, on the dynamic range ratio, on the near field properties and on the pattern reconfigurability.

For testing the array performance in a realistic scenario, the open-source electromagnetic simulator gprMax [16] may be employed, implementing the Finite-Difference TimeDomain method.

\section{Acknowledgements}

This work is a contribution to COST (European COoperation in Science and Technology, www.cost.eu) Action TU1208 "Civil engineering applications of Ground Penetrating Radar" (www.GPRadar.eu). The authors thank COST for funding and supporting the Action TU1208.

\section{References}

[1] A. Benedetto and L. Pajewski, Eds., Civil Engineering Applications of Ground Penetrating Radar, Book Series: Springer Transactions in Civil and Environmental Engineering. Springer, 2015 (doi: 10.1007/978-3-319-04813-0).

[2] R. Persico, Introduction to Ground Penetrating Radar: Inverse Scattering and Data Processing. Wiley-IEEE Press, 2014.

[3] F. Tosti, S. Adabi, L. Pajewski, G. Schettini, and A. Benedetto, "Large-scale analysis of dielectric and mechanical properties of pavement using GPR and LFWD", in Proc. 15th Int. Conf. on Ground Penetrat. Radar GPR 2014, Brussels, Belgium, 2014, pp. 868-873 (doi: 10.1109/ICGPR.2014.6970551).

[4] E. Huuskonen-Snicker, P. Eskelinen, T. Pellinen, and M.-K. Olkkonen, "A new microwave asphalt radar rover for thin surface civil engineering applications", Frequenz J. of RF-Engin. and Telecommun., vol. 69, no. 7-8, pp. 377-381, 2015 (doi: 10.1515/freq-2015-0034).

[5] R. Persico and G. Leucci, "Interference mitigation achieved with a reconfigurable stepped-frequency GPR system", Remote Sensing, vol. 8, no. 11, Article no. 926, 2016 (doi: 10.3390/rs8110926).

[6] V. Ferrara, F. Troiani, F. Frezza, F. Mangini, L. Pajewski, P. Simeoni, and N. Tedeschi, "Design and realization of a cheap Ground Penetrating Radar prototype @ $2.45 \mathrm{GHz}$ ", in Proc. 10th IEEE Eur. Conf. on Antenn. and Propag. EuCAP 2016, Davos, Switzerland, 2016, pp. 1-4 (doi: 10.1109/EuCAP.2016.7482008).

[7] L. Pajewski, R. Persico, S. Chicarella, V. Ferrara, F. Frezza, and F. Troiani, "Ground Penetrating Radar prototypes developed in COST Action TU1208", in Proc. 24th Int. Conf. on Software, Telecommun. and Comp. Netw. SoftCOM 2016, Split, Croatia, 2016, pp. 1-5.

[8] C. Warren, A. Giannopoulos, N. Diamanti, and P. Annan, "An extension module to embed commercially sensitive antenna models in gprMax", in Proc. 8th Int. Worksh. on Adv. Ground Penetrat. Radar IWAGPR 2015, Florence, Italy, 2015, pp. 1-3 (doi: 10.1109/IWAGPR.2015.7292623).

[9] C. Warren and A. Giannopoulos, "Experimental and modeled performance of a ground penetrating radar antenna in lossy dielectrics", IEEE J. of Selec. Topics in Appl. Earth Observ. and Remote Sensing (JSTARS), vol. 9, no. 1, pp. 29-36, 2016 (doi: 10.1109/JSTARS.2015.2430933). 
[10] E. Eide, P. A. Våland, and J. Sala, "Ground-coupled antenna array for step-frequency GPR", in Proc. 15th Int. Conf. on Ground Penetra. Radar GPR 2014, Brussels, Belgium, 2014, pp. 756-761 (doi: 10.1109/ICGPR.2014.6970527).

[11] L. Pajewski, F. Tosti, and W. Kusayanagi, "Antennas for GPR Systems", in Civil Engineering Applications of Ground Penetrating Radar, Book Series: Springer Transactions in Civil and Environmental Engineering, A. Benedetto and L. Pajewski, Eds. Springer, 2015, pp. 41-67 (doi: 10.1007/978-3-319-04813-0_2).

[12] S. Chicarella, V. Ferrara, F. Frezza, A. D’Alvano, and L. Pajewski, "Improvement of GPR tracking by using inertial and GPS combined data", in Proc. 24th Int. Conf. on Software, Telecommun. and Comp. Netw. SoftCOM 2016), Split, Croatia, 2016, pp. 1-5 (doi: 10.1109/SOFTCOM.2016.7772148).

[13] R. Vescovo, L. Pajewski, and F. Tosti, "State-of-the-art and trends of Ground-Penetrating Radar antenna arrays", Geophysical Research Abstracts, European Geosciences Union (EGU) General Assembly 2016, article ID EGU2016-18097, 2016, Vienna, Austria.

[14] G. Buttazzoni and R. Vescovo, "An efficient and versatile technique for the synthesis of 3D copolar and crosspolar patterns of phaseonly reconfigurable conformal arrays with DRR and near-field control", IEEE Trans. on Antenn. and Propag., vol. 62, no. 4, part 1, pp. 1640-1651, 2014.

[15] B. Fuchs, "Synthesis of sparse arrays with focused or shaped beampattern via sequential convex optimizations", IEEE Trans. on Antenn. and Propag., vol. 60, no. 7, pp. 3499-3503, 2012.

[16] C. Warren, A. Giannopoulos, and I. Giannakis, "gprMax: Open source software to simulate electromagnetic wave propagation for Ground Penetrating Radar", Comp. Phys. Commun., vol. 209, pp. 163-170 (doi: 10.1016/j.cpc.2016.08.020).

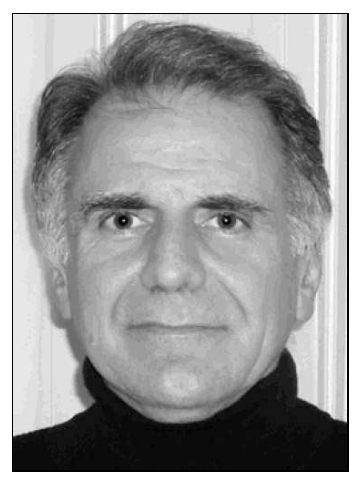

Roberto Vescovo received the Laurea degree (summa cum laude) in Electronic Engineering from the University of Trieste, Italy (1982), and the Ph.D. in Electronics and Information Engineering from the University of Padova, Italy (1987). He served as an Assistant Professor at the Department of Engineering and Architecture, University of Trieste, where he is currently an Associate Professor of electromagnetic fields. His main research interests are the synthesis of antenna arrays, the electromagnetic scattering, the electromagnetic theory and numerical methods, and the study of interactions between electromagnetic fields and relativistic electrons.

E-mail: vescovo@units.it

Department of Engineering and Architecture University of Trieste

via A. Valerio 10

34127 Trieste, Italy

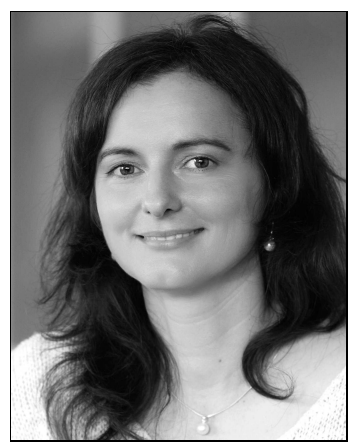

Lara Pajewski is a Professor of electromagnetic fields in the Department of Information Engineering, Electronics and Telecommunications of Sapienza University of Rome, Italy. She is an electronic engineer with a Ph.D. in applied electromagnetics and electrophysics sciences earned in Sapienza University. Since 2013, she is chairing the COST Action TU1208 "Civil engineering applications of Ground Penetrating Radar". Her current research interests include Ground Penetrating Radar (GPR) technology, methodology and applications, integration of GPR with complementary non-destructive testing methods, electromagnetic modeling of complex scenarios, antenna design and characterization, measurement of electromagnetic pollution and evaluation of its impact.

E-mail: lara.pajewski@uniroma1.it

Department of Information Engineering, Electronics and Telecommunications

Sapienza University of Rome

via Eudossiana 18

00184 Rome, Italy 